\title{
A method for the assessment of time-varying brain shift during navigated epilepsy surgery
}

\author{
E. De Momi ${ }^{1}$ - G. Ferrigno ${ }^{1}$ - G. Bosoni ${ }^{1}$ - P. Bassanini ${ }^{1}$ - P. Blasi ${ }^{1}$ - G. Casaceli $^{2}$ \\ D. Fuschillo ${ }^{2}$ - L. Castana ${ }^{2}$ - M. Cossu' ${ }^{2}$ - G. Lo Russo ${ }^{2}$ - F. Cardinale ${ }^{2}$
}

Received: 8 January 2015 / Accepted: 1 July 2015 / Published online: 17 July 2015

(C) CARS 2015

\begin{abstract}
Purpose Image guidance is widely used in neurosurgery. Tracking systems (neuronavigators) allow registering the preoperative image space to the surgical space. The localization accuracy is influenced by technical and clinical factors, such as brain shift. This paper aims at providing quantitative measure of the time-varying brain shift during open epilepsy surgery, and at measuring the pattern of brain deformation with respect to three potentially meaningful parameters: craniotomy area, craniotomy orientation and gravity vector direction in the images reference frame.

Methods We integrated an image-guided surgery system with 3D Slicer, an open-source package freely available in the Internet. We identified the preoperative position of several cortical features in the image space of 12 patients, inspecting both the multiplanar and the $3 \mathrm{D}$ reconstructions. We subsequently repeatedly tracked their position in the surgical space. Therefore, we measured the cortical shift, following its time-related changes and estimating its correlation with gravity and craniotomy normal directions.

Results The mean of the median brain shift amount is $9.64 \mathrm{~mm}(\mathrm{SD}=4.34 \mathrm{~mm})$. The brain shift amount resulted not correlated with respect to the gravity direction, the craniotomy normal, the angle between the gravity and the craniotomy normal and the craniotomy area.

Conclusions Our method, which relies on cortex surface 3D measurements, gave results, which are consistent with liter-
\end{abstract}

$凶$ E. De Momi

elena.demomi@polimi.it

1 Department of Electronics, Information and Bioengineering (DEIB), Politecnico di Milano, Piazza Leonardo da Vinci, 32, 20133 Milan, Italy

2 “Claudio Munari” Centre for Epilepsy and Parkinson Surgery A.O. Ospedale Niguarda Ca' Granda, Milan, Italy ature. Our measurements are useful for the neurosurgeon, since they provide a continuous monitoring of the intraoperative sinking or bulking of the brain, giving an estimate of the preoperative images validity versus time.

Keywords Brain shift · Epilepsy surgery · Frameless stereotaxy · Image-guided neurosurgery · Neuronavigation

\section{Introduction}

Image-guided neurosurgery is used to intra-operatively determine the best access to the surgical field and to choose the best pathway toward the target. Registration between the image space and the surgical space is the preliminary step, and it can be accomplished by identifying corresponding landmarks or surfaces [1,2]. Image guidance can support the surgeon during many types of surgery, such as resections aimed at the removal of brain tumors or of epileptogenic zones.

The intrinsic accuracy of frameless stereotactic systems ranges between 1 and $2 \mathrm{~mm}$. The localization accuracy during craniotomy surgery is mainly influenced by the severe shift of the brain $[3,4]$. Brain shift occurs once the dura is opened and before the interventional procedure is actually started: Once the actual procedure has started, the parenchyma may have consistently changed with respect to preoperative imaging exams (Fig. 1). The brain shift is mainly due to cerebrospinal fluid drainage, pharmaceuticals administered during the intervention and tissue resection [4]. A compensation for, or at least an assessment of, the brain shift is therefore essential and can be accomplished by performing intra-operative scans of the patient [5] or including accurate brain computational models which deform during time [6].

Brain shift amount has been estimated measuring landmarks on the cortex, by tracking devices (direct measure- 

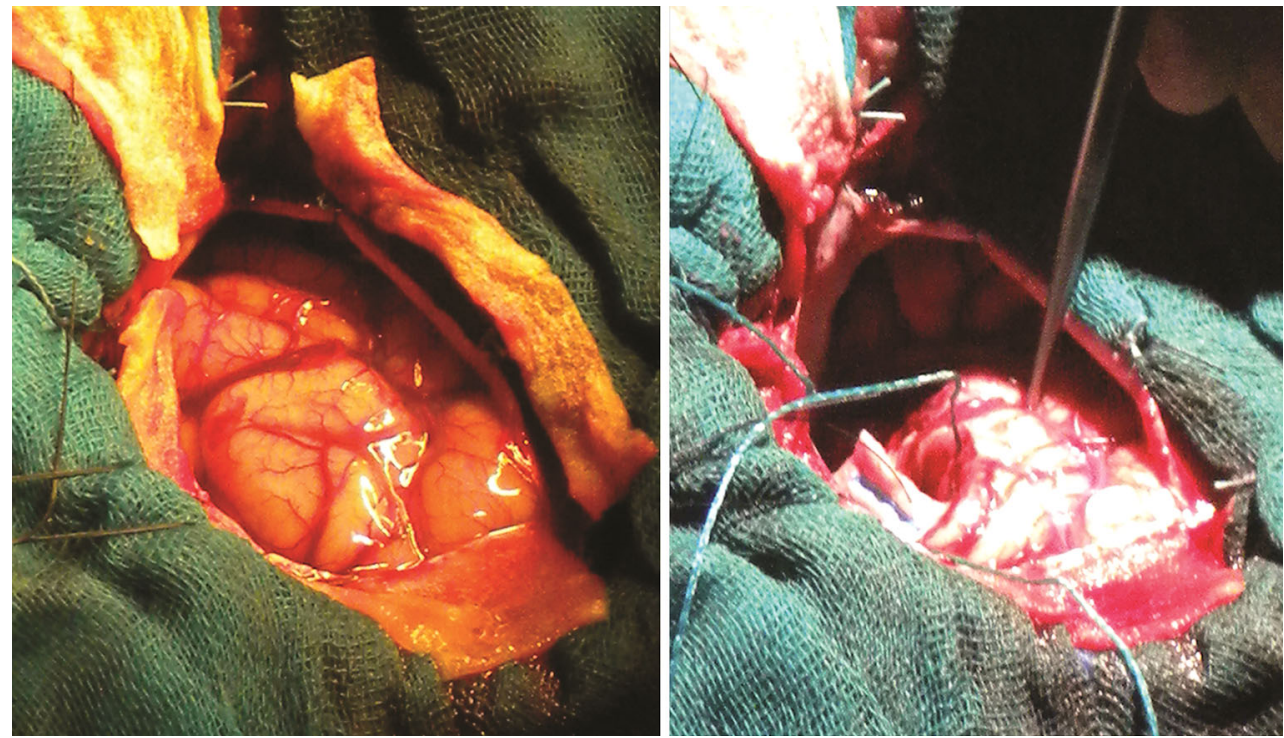

Fig. 1 On the left, exposed brain cortex surface right after dura opening. On the right, collapsed brain surface during the intervention

ments), with preoperative magnetic resonance (MR) image data [4] or using intra-operative images (indirect measurements), such as MR [5-7] and ultrasound (US) [8].

To our present knowledge, the work done by Roberts and colleagues [9] was the first to set the ground for brain shift measurements in a repeatable and imaging-free fashion: The surgeon manually pointed to pre-defined cortical features and acquired their position by means of a navigated surgical microscope exploiting the focus system.

In case of direct measurements on landmarks, reported mean shift of the cortex values are around $5-10 \mathrm{~mm}$ and maximum shift over $20 \mathrm{~mm}$ [10]. Cortical surface can also be acquired by stereoscopic [11] or laser range scanning approaches $[12,13]$.

Intra-operative imaging modalities provide information also on the shift of deep brain structures [14], which is small compared to cortex displacements. Mean cortical shifts of $5-6 \mathrm{~mm}$ have been reported, with maximum shift of over $20 \mathrm{~mm}$, and mean tumor shifts of 3-7 mm (this really depends on the position and nature of the tumor), with a maximum of $15 \mathrm{~mm}$. Unfortunately, interventional MR (iMR) scanners are expensive and can limit the access of the surgeon to the operating field. Intra-operative US, although more and more widespread, produces images at low resolution and low signal-to-noise ratio with poor anatomical detail. On top of this, both US-probe tracking accuracy and US image plane calibration accuracy influence the exact 3D localization of anatomical structures [15].

Recent studies have estimated brain shift in order to update the preoperative images using brain biomechani- cal models, which are deformed according to some source terms, such as the direction of gravity and the size of resection $[13,16]$. Once the model is computed, full volumetric displacements are available for updating preoperative images according to the intra-operative deformations of the brain structures. Indeed, very accurate biomechanical models, including detailed anatomical structures [17], require high computational time and intra-operative data retrieval $[13,15,18]$. Joldes [19] presented an efficient computational method for estimating intra-operative organ deformations, but they validated the approach only in simulations.

Moreover, studies investigating the laws of brain deformation, such as the one published by Hartkens [8], reported that the pattern of brain deformation, assessed in terms of magnitude of the shift, direction of displacement and change of volume, is extremely complex and not always correlated with gravity direction. Therefore, biomechanical models that use limited intra-operative information are unlikely to predict brain deformation for all cases. Furthermore, mechanical properties of the tissue, which are patient-dependent, should be well known in advance for estimation.

In this frame, the aim of this study was to present a novel approach to the problem of estimating the brain shift, using preoperative images, a neuronavigator and a personal computer. We developed a technique to estimate the amount of brain shift that occurs just after the opening of the dura mater, investigated the amount of time-varying brain shift during surgery and the dependence of brain deformation with respect to craniotomy area, craniotomy orientation and gravity direction. 


\section{Materials and methods}

\section{Preoperative data acquisition and processing}

Twelve drug-resistant epileptic patients (aged $32 \pm 14.63$ years) were included in this study, as shown in Table 1 . Data were acquired at the "Claudio Munari" Centre for Epilepsy and Parkinson Surgery, Ospedale Niguarda Ca' Granda, Milan, Italy. The study was approved by the hospital's ethical committee. All patients or their parents/guardians signed an informed consent.

T1-weighted 3D fast-field echo data were acquired (1.5T, Intera Achieva, Philips Medical System, the Netherlands) as sagittal images, $0.90 \mathrm{~mm} \times 0.45 \mathrm{~mm} \times 0.45 \mathrm{~mm}$ voxel dimensions, without any inter-slice gap; images were then reconstructed and subsequently reformatted on the axial plane directly on the MR scanner console so that axial images, $560 \times 560 \times 220$ voxels $(0.45 \mathrm{~mm} \times 0.45 \mathrm{~mm} \times$ $0.9 \mathrm{~mm}$ ), were obtained for the following clinical use and processing. No landmarks were fixed to the head.

Three-dimensional MRI datasets were processed with FreeSurfer (http://surfer.nmr.mgh.harvard.edu/) [20] in order to segment brain structures and to reconstruct a model of the brain surface. A patient-specific model of the pial surface was therefore generated for each patient. All the processed output datasets were loaded into the open-source 3D Slicer platform (Slicer 4.3.1, www.slicer.org/y) [21] for visualization. As needed, other image datasets, such as fluidattenuated inversion recovery MR scans (FLAIR), diffusion tensor imaging (DTI), functional magnetic resonance imaging (fMRI) or positron emission tomography (PET), were included in the multimodal scene. This image processing workflow is routinely performed at the "Claudio Munari" Centre [22-26].

Table 1 Patients' data: age at the time of the intervention, gender and procedure type are reported

\begin{tabular}{lrll}
\hline & Age & Gender & Procedure type \\
\hline 1 & 30 & M & Left temporal antero-mesial lobectomy \\
2 & 13 & M & Left temporal antero-mesial lobectomy \\
3 & 9 & M & Right supramarginal gyrus resection \\
4 & 32 & F & Left frontal resection \\
5 & 24 & M & Left temporal antero-mesial lobectomy \\
6 & 51 & M & Left temporal antero-mesial lobectomy \\
7 & 15 & M & Left temporal antero-lateral resection \\
8 & 41 & M & Left frontal resection \\
9 & 33 & M & Left temporal antero-mesial lobectomy \\
10 & 44 & M & Left temporal antero-mesial lobectomy \\
11 & 54 & M & Left temporal antero-mesial lobectomy \\
12 & 38 & M & Right temporal antero-mesial lobectomy \\
\hline
\end{tabular}

\section{Intra-operative navigation}

In our center, the patient's head is fixed using a 3-pin Mayfield skull clamp and registered matching the skin surface with its reconstruction available in the workstation of the navigation system (StealthStation ${ }^{\circledR}$ TREON Plus, Medtronic, Inc., Louisville, CO, USA). For the purposes of the study, the navigator was connected via a network communication interface (StealthLink ${ }^{\circledR}$, Medtronic, Inc., Louisville, CO, USA) to 3D Slicer environment, running on an Apple Mac Pro (Mac OS $X$ 10.8) (Fig. 2). Two reference frames (RF) are solid with the Mayfield clamp $\left(\mathrm{RF}_{\mathrm{P}}\right)$ and a pointer probe $\left(\mathrm{RF}_{\mathrm{Pr}}\right)$ with passive markers, which are tracked by the optical localization system (Fig. 3). The transformation from the images space $R F_{I}$ to the surgical space $R F_{P}$ is sampled (at $60 \mathrm{~Hz}$ ) and sent to a Windows client using the Ethernet communication channel and the StealthLink ${ }^{\circledR}$ protocol. Transformations are then sent to the 3D Slicer environment using OpenIGTLink [27], an open-source network protocol specifically developed for operating room devices communication.

All the 12 patients were in supine position with the head tilted contralaterally to the resection site. Intraoperatively, none of the patients received any treatment to reduce intracranial pressure (ICP).

\section{Landmarks acquisition protocol}

Landmarks acquisition protocol was performed in two different steps: intra-operative and preoperative.

(1) Intra-operatively, after the craniotomy was performed, $M_{i}$ points with coordinates $C_{M_{i}}$ were acquired on the craniotomy edges (where $i=1 \ldots 12$ indicates the patient number), as shown in Fig. 4, as evenly and uniformly as possible.

After the dura was opened, $N_{i}$ landmarks (with coordinates $L_{N_{i}}$ ) were selected by the neurosurgeons (Fig. 4). Those landmarks were points easily identifiable by the neurosurgeon during the entire operation, such as vessel bifurcations or sterile paper disks positioned on the cortical surface. These latter are used currently during the surgical intervention in order to get oriented. They are in fact positioned on the scars of the SEEG electrodes, which are well identified in the plan images. Such points were positioned externally to the resection area as spread as possible. Points were acquired using the optically tracked probe pointer at $t_{1}$ (immediately after the dura opening), at $t_{2}$ (estimated to be the half of the resection by the surgeon, if possible) and at $t_{3}$ (immediately before dura closure).

Acquisition time was also recorded and referred to 0 , corresponding to the dura opening.

(2) Landmarks position before surgery $\left(t_{0}\right)$ was a posteriori identified on the pial surface model. The surgeon 

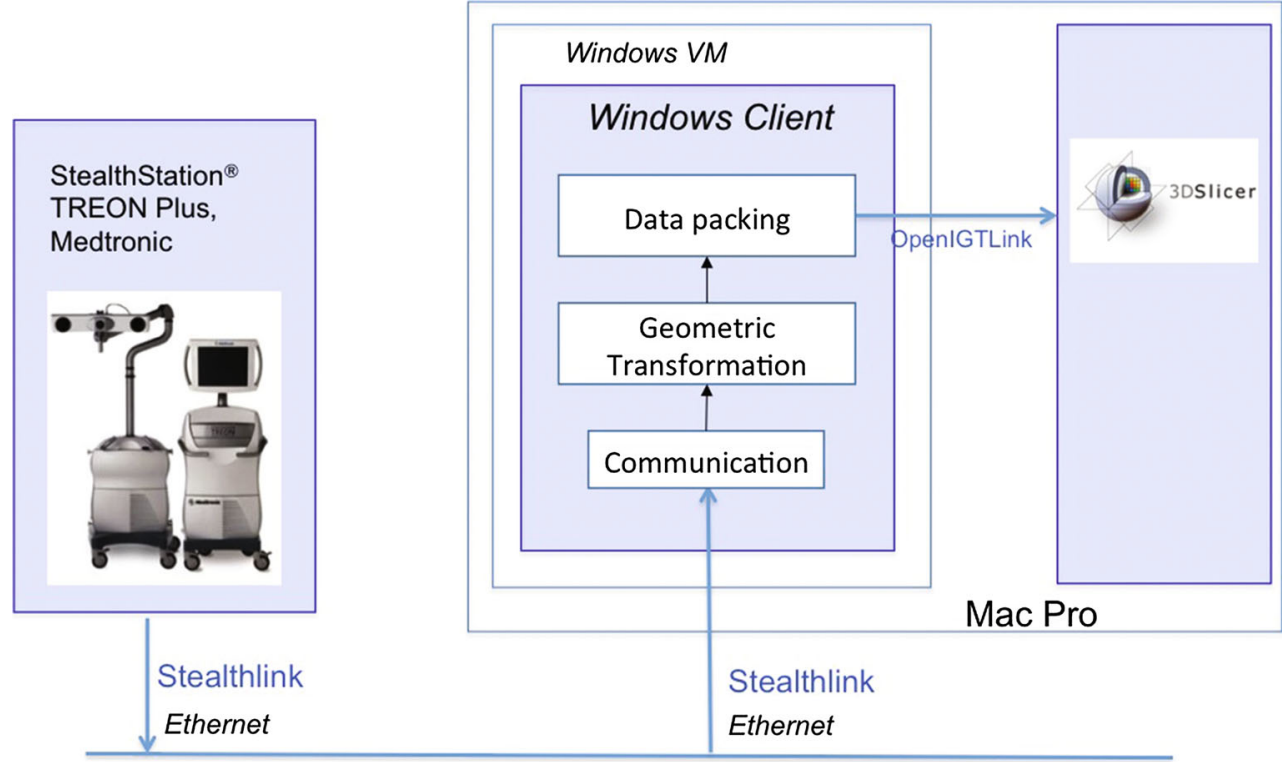

\section{Hospital network}

Fig. 2 System architecture and communication protocol. The StealthStation ${ }^{\circledR}$ TREON Plus is connected via Ethernet interface to the Mac Pro using the StealthLink protocol. A Windows client, running on a
Windows 7 virtual machine, retrieves data from the StealthStation. Data are transferred to the 3D Slicer environment using the OpenIGTLink protocol

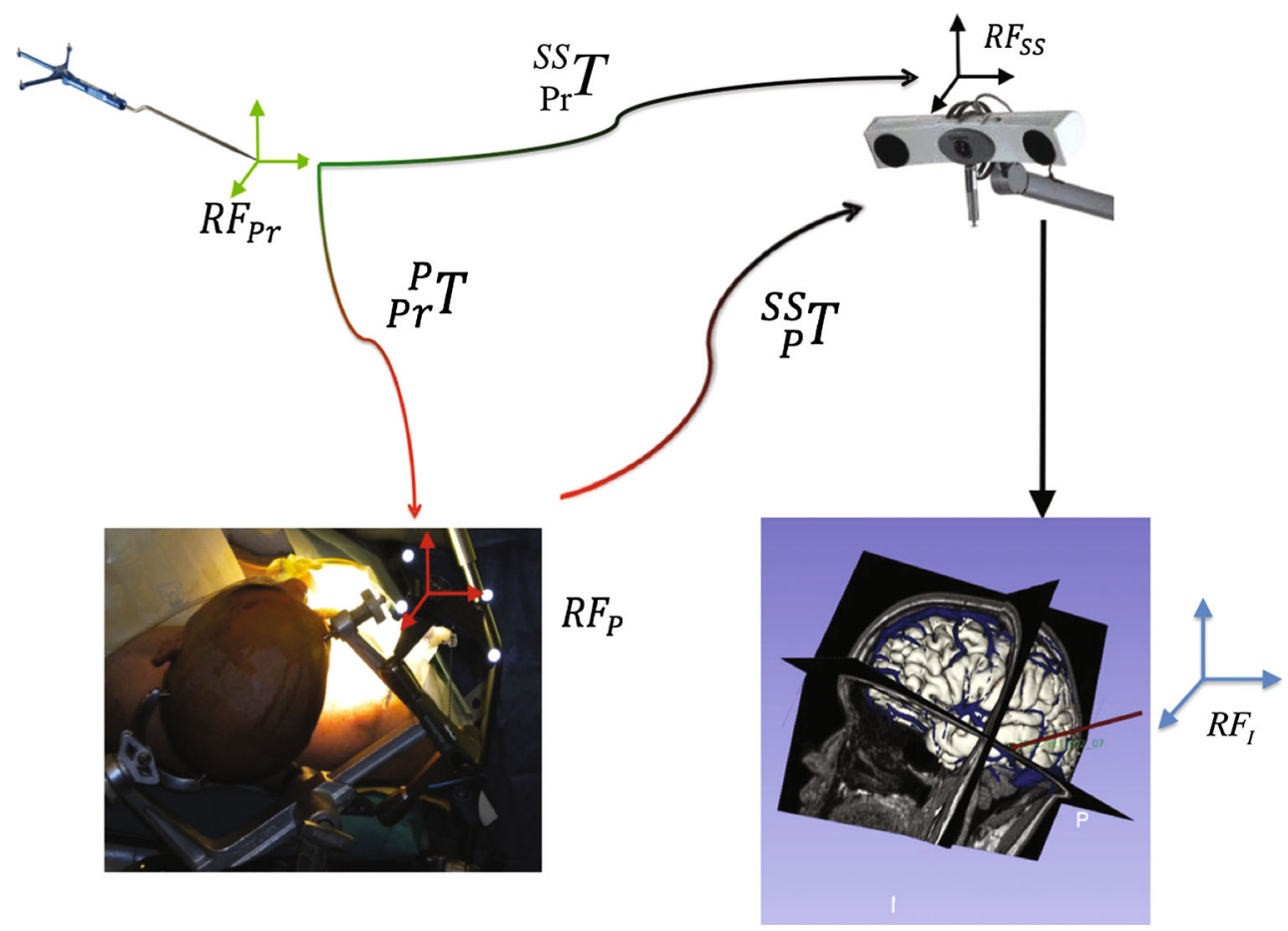

Fig. 3 Geometrical transformations between the optical localization system of the StealthStation $\left(\mathrm{RF}_{\mathrm{SS}}\right)$, the intra-operative environment $\left(\mathrm{RF}_{\mathrm{P}}\right)$ and the 3D Slicer environment $\left(\mathrm{RF}_{\mathrm{I}}\right)$

manually selected the landmarks on patient's 3D Slicer scene after recognizing sulcal and vascular bifurcations as anatomical features. This task was carried out after the resection, since before the surgeon cannot know which feature will be clearly visible after opening. Anyway, it is done on preoperative images and thus represents correctly time $t_{0}$. 

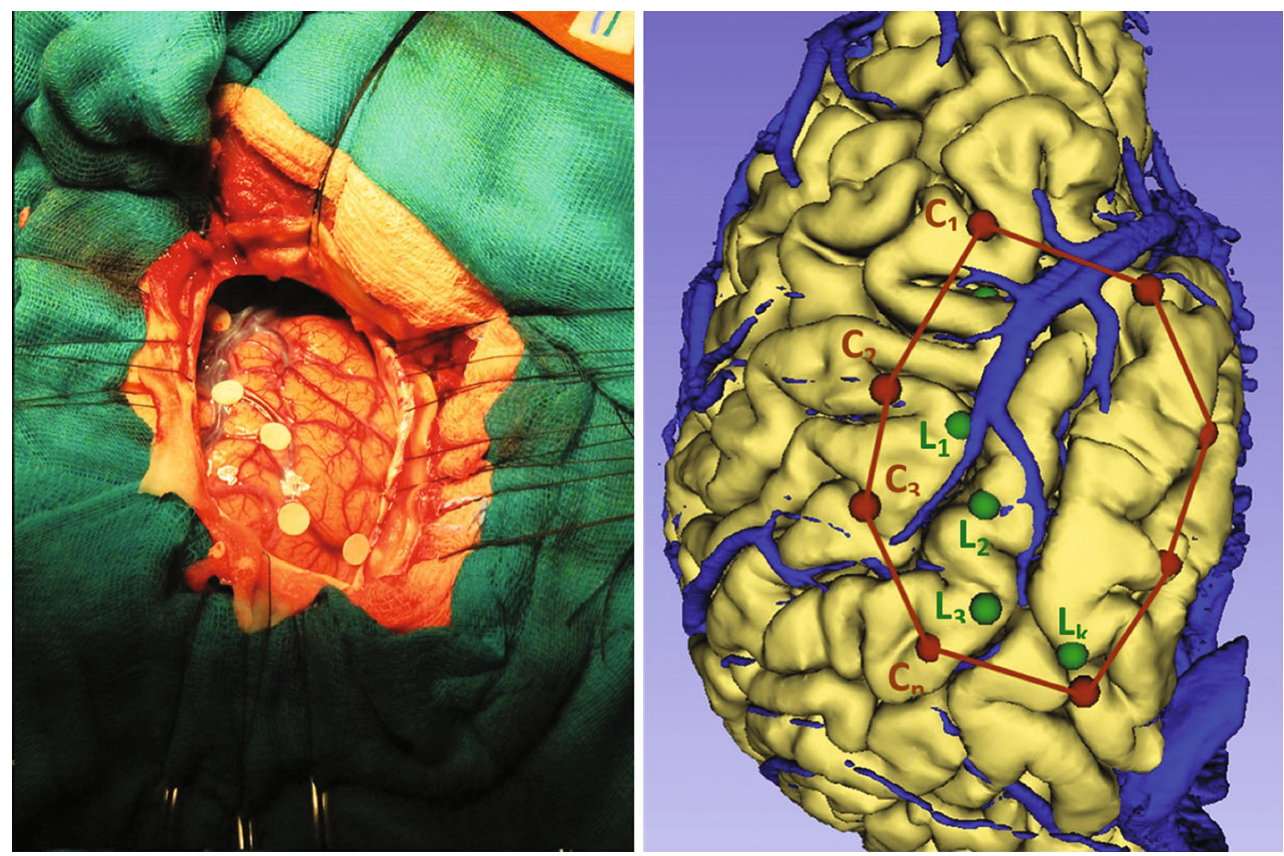

Fig. 4 Acquired points at $t 1\left(L_{N_{i}}\right)$ are showed: in this case, on the left, sterile paper landmarks positioned on the cortical surface are represented, on the right, by the green spheres on 3D Slicer model. Points $C_{M_{i}}$ are positioned on the craniotomy rim margins

\section{Data processing and brain shift estimation}

Manual acquisition repeatability was evaluated, asking the neurosurgeon participating to the experiment to repeat the acquisition of the same points twice within some seconds and calculating the norm of the difference between the points acquired position. More than two acquisitions were not reckoned compatible with surgical workflow (excessive time burden).

For each patient, the craniotomy area $\left(A_{i}\right)$ was estimated as the area of the polygonal shape resulting from the acquired $C_{M_{i}}$ fiducials. Also, the direction (normal versor) of the plane fitting the craniotomy points $\left(\mathbf{c}_{\mathbf{n}}\right)$ was computed. The gravity direction $(\mathbf{g})$ in the image reference frame was acquired using two vertical points on a photographic tripod whose bar was set vertical using a bull's eye spirit level.

We indicate the estimated brain shift at time interval $k-$ $1, k$ as $\mathrm{BS}\left({ }_{k}^{k-1} T\right), k=1, \ldots, 3$. Corresponding point set registration matching [28] was used to estimate the $4 \times 4$ homogeneous matrix $\mathrm{T}$ that maps the acquired points at time $k-1$ to time $k$, using the two sets of points acquired in subsequent times $k-1$ and $k$ : in particular, $T$ was computed starting from the estimate of the quaternion representing the transformation. The linear fitting was chosen since the points are collected on a small cerebral exposed surface; therefore, we hypothesized that the displacement occurred on that brain portion can be considered rigid (first order approximation). The computed spatial transformation is thus reliable only in a limited portion of the brain surface. The brain shift (indicated by $\mathrm{BS}_{k-1, k}$ ) was defined as the norm of the translation vector of the transformation matrix among groups of corresponding points (indicated by $\mathbf{b s}_{k-1, k}$ ):

$\mathrm{BS} k-1, k=\left\|\mathbf{b s}_{k-1, k}\left(k_{k}^{k-1} T\right)\right\|, k=1, \ldots, 3$

For each time instant, we also computed $\alpha_{k-1, k}$ as the angle between vector $\mathbf{b s}_{k-1, k}$ and the gravity direction in images reference frame $\mathbf{g}$ and $\gamma_{k-1, k}$ as the angle between vector $\mathbf{b s}_{k-1, k}$ and the craniotomy normal direction $\mathbf{c}_{\mathbf{n}}$.

The Pearson correlation indexes $(c)$ of $\mathrm{BS}_{0,3}$ with respect to:

- gravity direction in images reference frame $\alpha_{k-1, k}$

- craniotomy normal direction $\gamma_{k-1, k}$

- angle $\beta$ between craniotomy normal $\mathbf{c}_{\mathbf{n}}$ and gravity $\mathbf{g}$ (Fig. 5)

- craniotomy area $A$

were computed $(p<0.05)$.

Statistical analysis was performed using R statistical software (version 3.0.2) [29].

\section{Results}

Acquisition repeatability on two acquisitions was $1.15 \mathrm{~mm}$ (median value) with quantiles $q_{25} \%=0.75 \mathrm{~mm}$ and $q_{75} \%=$ $1.72 \mathrm{~mm}$. 


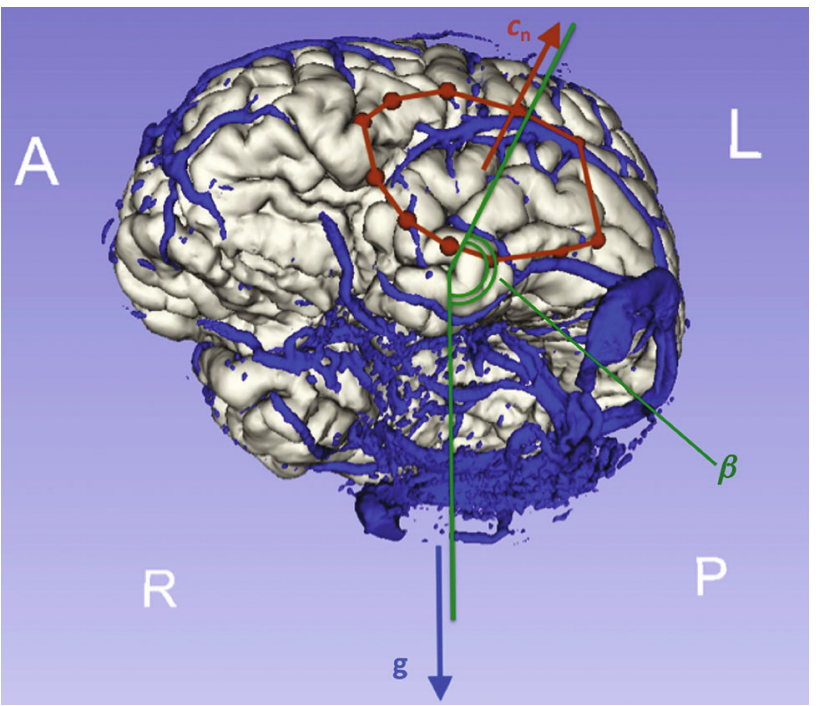

Fig. 5 Craniotomy landmarks $\left(C_{M_{i}}\right)$, craniotomy normal $\left(\mathbf{c}_{\mathbf{n}}\right)$ and gravity $(\mathbf{g})$ vectors are shown along with the angle between them $(\beta)$

Table 2 shows the craniotomy areas $\left(q_{50 \%}=2332.75 \mathrm{~mm}^{2}\right.$, $q_{25 \%}=2107.62 \mathrm{~mm}^{2}, q_{75 \%}=2552.12 \mathrm{~mm}^{2}$ ) of the patients. Generally, the craniotomy was ellipsoid in shape. The variability of the angle between craniotomy normal $\mathbf{c}_{\mathbf{n}}$ and gravity $\mathbf{g}(\beta)$ is low $\left(136.13^{\circ} \pm 4.16^{\circ}\right)$.

Figure 6 shows the BS with respect to the acquisition time for each of the 12 patients. The BS plot has a similar trend in all patients: BS is greater at the beginning of the procedure (4-15 mm). As reported in Fig. 7, the mean of the median $\mathrm{BS}_{0,3}$ is $9.64 \mathrm{~mm}(\mathrm{SD}=4.34 \mathrm{~mm})$.

There is not any significant correlation in the variations of the angle between the brain shift vector (bs) and the gravity $(\alpha)$ over time $(p=0.96)$ nor of the angle between bs and the normal to the craniotomy plane $(\gamma)$ over time $(p=0.43)$. We also found a great variability in $\alpha$ in every patient, since

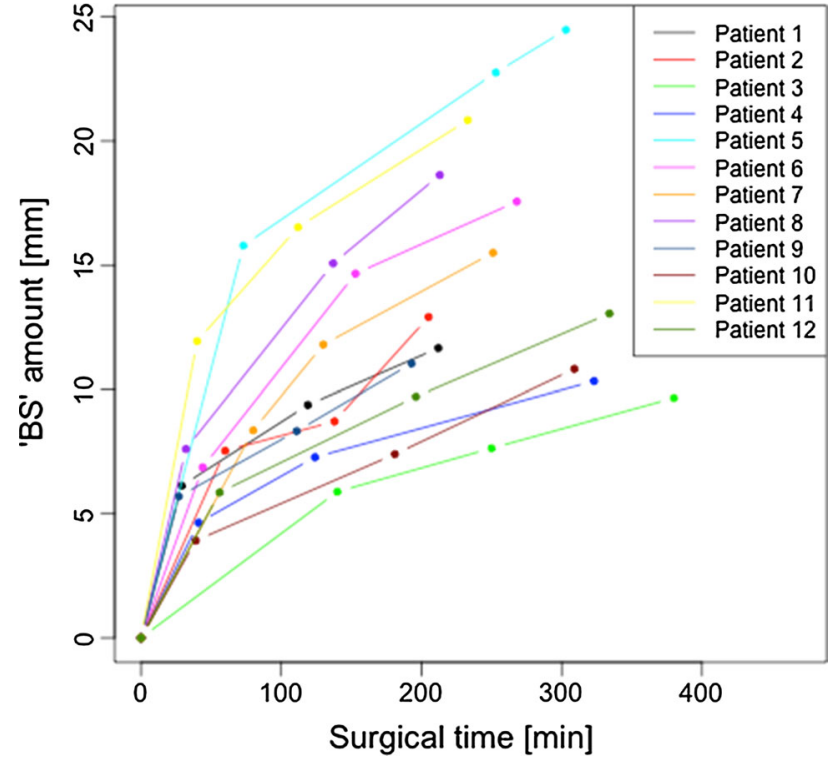

Fig. $6 \mathrm{BS}$ for each patient with respect to the acquisition time $\left(0, t_{1}, t_{2}\right.$ and $\left.t_{3}\right): \mathrm{BS}_{0,1}, \mathrm{BS}_{1,2}, \mathrm{BS}_{2,3}$

the mean value, considering the whole intervention duration $\left(\alpha_{0,3}\right)$, is $33.05^{\circ}\left( \pm 19.14^{\circ}\right)$.

Table 3 reports the correlation coefficients of the total amount of brain shift $\left(\mathrm{BS}_{0,3}\right)$ with respect to the gravity direction, the craniotomy normal, the angle between the gravity and the craniotomy normal and the craniotomy area. Generally, the brain shift is not correlated with any of these parameters.

\section{Discussion}

We report the analysis of brain landmarks displacement during navigated interventions for drug-resistant epilepsy
Table 2 Patients' data: craniotomy areas and number of landmarks acquired for each patient

\begin{tabular}{lllll}
\hline Patient number & $\begin{array}{l}\text { Craniotomy } \\
\text { area }\left(\mathrm{mm}^{2}\right) A\end{array}$ & Angle $\beta$ & $\begin{array}{l}\text { \# of acquired } \\
\text { landmarks } N\end{array}$ & $\begin{array}{l}\text { \# of } \\
\text { acquisitions }\end{array}$ \\
\hline 1 & 2448.94 & 135.42 & 5 \\
2 & 2462.47 & 132.2 & 5 \\
3 & 1949.92 & 133.36 & 6 \\
4 & 5951.63 & 133.59 & 4 & \\
5 & 1861.75 & 136.01 & 5 & \\
6 & 2283.84 & 139.03 & 5 & \\
7 & 2058.33 & 142.18 & 5 \\
8 & 4190.88 & 144.1 & 5 \\
9 & 2381.66 & 131.08 & 6 \\
10 & 2153.12 & 131.89 & 5 \\
11 & 2124.05 & 138.95 & 4 \\
12 & 2821.08 & 135.76 & 4 \\
\hline
\end{tabular}




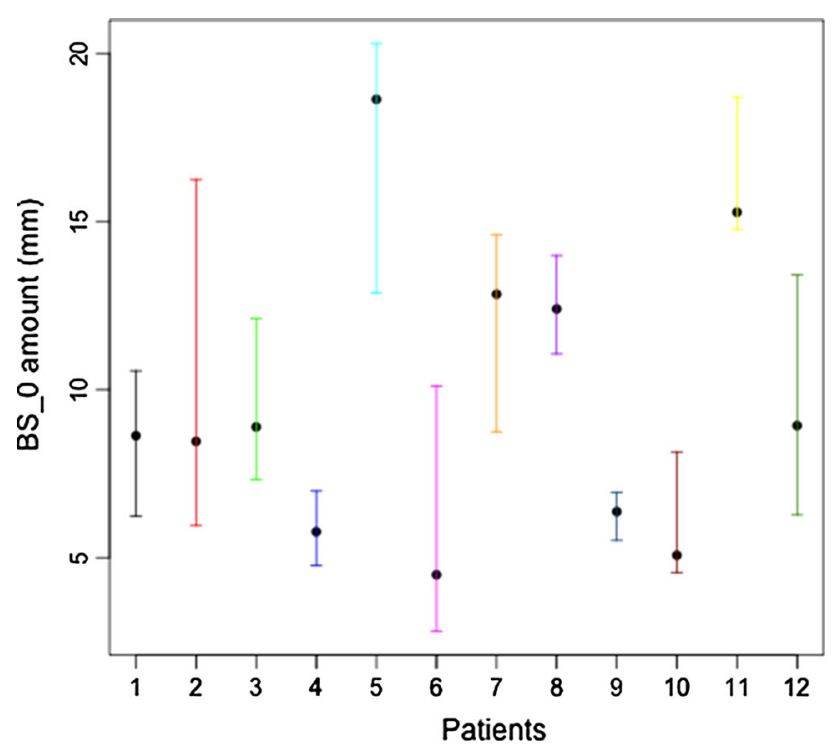

Fig. $7 \mathrm{BS}_{0,3}$ amount for each patient

surgery. The aim of the study was to quantify the brain shift during open skull interventions, sampling the brain cortex three times during the surgical intervention, from the dura opening until the dura closing, and relating those measures to the estimated preoperative position of the selected anatomical features. Landmarks were selected using an optical pointer and a commercial navigation system, adapted and integrated into the 3D Slicer environment. Landmarks selection repeatability was measured and turned out to be in the same order of magnitude of the navigation system registration error, which is reported to be $<1 \mathrm{~mm}$ [30]. According to previous studies, the possibility of performing repeated measurements on the same points is a benefit, since it allows monitoring of the brain surface changes over time [31].

First, we point out that the low variability of $\beta$ is mainly because 10 out of 12 patients considered in this study underwent temporal resection and head placement in this kind of procedure is standard.

In this context, we measured a mean of the median total $\mathrm{BS}$ amount of $9.64 \mathrm{~mm}(\mathrm{SD}=4.34 \mathrm{~mm})$, while as reported in [12], amplitude of deformations can exceed $20 \mathrm{~mm}$. The analysis also shows that deformations are not uniform among patients and, within a patient, over time; furthermore, their direction is not always close to the gravity direction.

Letterboer and colleagues [12] assert that the shift prior to opening the dura mater was larger than the shift introduced by opening it. On the other hand, Sun hypothesized a minimal brain deformation before the dural opening [11], but they did not perform any measurement. We cannot support one or the other, since the measurements are taken with either intact skull or open dura, but our results confirm that brain shift velocity is not constant in time. Our findings demonstrate a big shift immediately after the dura is opened and a following reduction in shift rate. Even though we are not able to quantify how much of the shift occurs before the dura is opened and how much takes place after that, as done in [12], we are able to show brain shift trend throughout the whole procedure. It must be noted that the brains of the epileptic patients are morphologically normal, so a small shift should be expected at dura opening (due to normal ICP). In addition to this, having not taken advantage of any measure to reduce ICP (like hyperventilation or diuretic drug administration), we can actually ascribe the amount of brain shift measured to the procedure alone.

On the other hand, this sets a range of validity for our study: In fact, it has to be limited only to procedures involving patients whose ICP is not elevated by any pathologic condition (requiring diuretics or hyperventilation for management) and thus require pharmaceutical management.

Letterboer and colleagues [12] also report that the angle between gravity and the main direction of shift is $60^{\circ}$ on average, with a maximum of $88^{\circ}$. In our 12 patients, the angle with respect to gravity is smaller $\left(33.05^{\circ}\right.$ as average value, $55.18^{\circ}$ as maximum) but with a wide variability range ( $19.14^{\circ}$ being the standard deviation). This value depends on the craniotomy normal direction, which is related to the surgical target zone and to each surgical team habits. Generally, we did not assess any correlation of the BS with respect to the gravity direction, the craniotomy normal, the angle between the gravity and the craniotomy normal or the craniotomy area. There is a positive correlation (but not statistically significant, $p=0.12$ ) between the $\mathrm{BS}$ and $\beta$, meaning that the bigger is that angle, the bigger is the BS. We hypothesize that this correlation could reach statistical significance widening the dimension of the sample under investigation.

In our study, the number of chosen corresponding landmarks was low and this is due to the difficulty identification of robust landmarks that are both far from the resection region and easily identifiable during the whole surgery. Furthermore, the sampling of the landmarks position during the surgical intervention was kept low to avoid interfering with the surgical procedure itself.
Table $3 \mathrm{BS}_{0,3}$ amount correlation coefficients

\begin{tabular}{lcccc}
\hline $\mathrm{BS}_{0,3}$ & Angle $\boldsymbol{\alpha}(\mathbf{b s}, \mathbf{g})$ & Angle $\boldsymbol{\gamma}\left(\mathbf{b s}, \mathbf{c}_{\mathbf{n}}\right)$ & Angle $\beta$ & Craniotomy area $\mathbf{A}$ \\
\hline$C$ & -0.02 & -0.25 & 0.48 & -0.09 \\
$p$ value & 0.96 & 0.43 & 0.12 & 0.79 \\
\hline
\end{tabular}


In conclusion, this study demonstrates the usefulness of monitoring the position changes undergone by the brain during open skull neurosurgical procedures in that, in principle, it allows the implementation of an automated routine concerned with updating the preoperative scans and informing the surgeon of the changes taking place in the operative field with respect to the surgical plan. In principle, feasibility can be achieved only without interfering with normal surgical workflow: In this regard, a less "invasive" way of acquiring cortical surface position is needed and can be realized using an automated surface segmentation algorithm, already under development in the ACTIVE [32] frame at Politecnico di Milano. This would also ensure a virtually real-time monitoring of the shift occurring to brain structures in proximity to the surface visible to the cameras, such as tumors, vessels, sulci and gyri [33].

It has also been shown that local volumetric brain deformation can be inferred given the cortical surface displacement [34]. In this context, inside the framework of the European project ACTIVE, a series of high-resolution model deformations based on brain constitutive law and complex loading conditions, such as brain shift due to gravity, organ volume changes due to drug reactions or due to edema, were developed. An ad hoc model could be preoperatively setup for each patient due to undergo epilepsy surgery, and these models could be used to construct an ad hoc "atlas" of deformations. Such atlas will be intra-operatively used to update the preoperative information on the surgical target position. In this way, the neurosurgical navigator, which cannot be considered reliable once the brain undergoes deformation subsequent to craniotomy, can still be used for navigating the intervention after reliable update of the images.

Acknowledgments The authors acknowledge the support of the FP7ICT-2009-6-270460 ACTIVE project. The authors also thank Daniele Marinucci and Medtronic (Minneapolis, MN, US) for allowing the use of StealthLink libraries for this specific work.

\section{Compliance with Ethical Standards}

Conflict of interest Francesco Cardinale serves as a paid consultant to Renishaw mayfield, the manufacturer of the Neuromate stereotactic robotic system (not mentioned in the paper). The other authors declare that they have no conflict of interest.

\section{References}

1. Schulz C, Waldeck S, Mauer UM (2012) Intraoperative image guidance in neurosurgery: development, current indications, and future trends. Radiol Res Pract 2012:1-9

2. Orringer DA, Golby A, Jolesz F (2012) Neuronavigation in the surgical management of brain tumors: current and future trends. Export Rev Med Devices 9(5):491-500

3. Hastreiter P, Rezk-Salama C, Soza G, Bauer M, Greiner G, Fahlbusch R, Ganslandt O, Nimsky C (2004) Strategies for brain shift evaluation. Med Image Anal 8(4):447-64
4. Hill DL, Maurer CR Jr, Maciunas RJ, Barwise JA, Fitzpatrick JM, Wang MY (1998) Measurement of intraoperative brain surface deformation under a craniotomy. Neurosurgery 43(3):514-528

5. Clatz O, Delingette H, Talos IF (2005) Robust nonrigid registration to capture brain shift from intraoperative MRI. IEEE Trans Med Imaging 24(11):1417-27

6. Valencia A, Blas B, Orega JH (2012) Modeling of brain shift phenomenon for different craniotomies and solid models. J Appl Math. doi:10.1155/2012/409127

7. Nimsky C, Ganslandt KA, Cerny S, Hastreiter P, Greiner G, Fahlbusch R (2000) Quantification of, visualization of, and compensation for brain shift using intraoperative magnetic resonance imaging. Neurosurgery 47(5):1070-1080

8. Hartkens T, Hill DLG, Castellano-Smith AD, Hawkes DJ, Maurer CR, Martin AJ, Hall WA, Liu H, Truwit CL (2003) Measurement and analysis of brain deformation during neurosurgery. IEEE Trans Med Imaging 22(1):82-92

9. Roberts DW, Hartov A, Kennedy FE, Miga MI, Paulsen KD (1998) Intraoperative brain shift and deformation: a quantitative analysis of cortical displacement in 28 cases. Neurosurgery 43(4):749-58

10. Kleary K, Peters TM (2010) Image-guided interventions: technology review and clinical applications. Annu Rev Biomed Eng 12:119-42

11. Sun H, Lunn KE, Farid H, Wu Z, Roberts DW, Hartov A, Paulsen KD (2005) Stereopsis-guided brain shift compensation. IEEE Trans Med Imaging 24(8):1039-52

12. Letterboer MM, Willems PW, Viergever MA, Niessen WJ (2005) Brain shift estimation in image-guided neurosurgery using 3D ultrasound. IEEE Trans Biomed Eng 52(2):268-296

13. Ding S, Miga MI, Thompson RC, Dumpuri P, Cao A, Dawant BM (2007) Estimation of intra-operative brain shift using a tracked laser range scanner. In: Engineering in Medicine and Biology Society, 2007. EMBS 2007. 29th annual international conference of the IEEE, pp 848-851. doi:10.1109/IEMBS.2007.4352423

14. Paulsen KD, Miga MI, Kennedy FE, Hoopes PJ, Hartov A, Roberts DW (2009) A computational model for tracking subsurface tissue deformation during stereotactic neurosurgery. IEEE Trans Biomed Eng 46(2):213-25

15. De Lorenzo D, Vaccarella A, Khreis G, Monnich H, Ferrigno G, De Momi E (2011) Accurate calibration method for 3D freehand ultrasound probe using virtual plane. Med Phys 38(12):6710-6720

16. Sinha TK, Dawant BM, Duay V, Cash DM, Weil RJ, Thompson RC, Weaver KD, Miga MI (2005) A method to track cortical surface deformations using a laser range scanner. IEEE Trans Med Imaging 24(6):767-81

17. Hu J, Jin X, Lee JB, Zhang L, Chaudhary V, Guthikonda M, Yang KH, King AI (2007) Intraoperative brain shift prediction using a 3D inhomogeneous patient-specific finite element model. J Neurosurg 106(1):164-9

18. Schaewe TJ, Fan X, Ji S, Hartov A, Hiemenz Holton L, Roberts DW, Paulsen KG, Simon DA (2013) Integration of intraoperative and model-updated images into an industry-standard neuronavigation system: initial results. In: Holmes DR, Yaniv ZR (eds) Medical imaging: image-guided procedures, robotic interventions, and modeling, SPIE 8671

19. Joldes GR, Wittek A, Miller K (2009) Computation of intraoperative brain shift using dynamic relaxation. Comput Methods Appl Mech Eng 198(41):3313-3320

20. Fischl B (2012) FreeSurfer. Neuroimage 62(2):774-781

21. Pieper S, Halle M, Kikinis R (2004) 3D SLICER. In: Proceedings of the IEEE international symposium on biomedical imaging, $\mathrm{pp}$ 632-635

22. De Momi E, Caborni C, Cardinale F, Castana L, Casaceli G, Cossu M, Antiga L, Ferrigno G (2013) Automatic trajectory planner for StereoElectroEncephaloGraphy procedures: a retrospective study. IEEE Trans Biomed Eng 60(4):986-93 
23. Cardinale F, Cossu M, Castana L, Casaceli G, Schiariti MP, Miserocchi A, Fuschillo D, Moscato A, Caborni C, Arnulfo G, Lo Russo G (2013) Stereoelectroencephalography: surgical methodology, safety, and stereotactic application accuracy in 500 procedures. Neurosurgery 72(3):353-66

24. Cardinale F, Miserocchi A, Moscato A, Cossu M, Castana L, Schiariti MP, Gozzo F, Pero G, Quilici L, Citterio A, Minella M, Torresin A, Lo Russo G (2012) Talairach methodology in the multimodal imaging and robotic era. In: Scarabin J (ed) Stereotaxy and epilepsy neurosurgery. John Libbey Eurotext, London, pp 245-272

25. Cardinale F, Chinnici G, Bramerio M, Mai R, Sartori I, Cossu M, Lo Russo G, Castana L, Colombo N, Caborni C, De Momi E, Ferrigno G (2014) Validation of FreeSurfer-estimated brain cortical thickness: comparison with histologic measurements. Neuroinformatics 12(4):535-42

26. De Momi E, Caborni C, Cardinale F, Casaceli G, Castana L, Cossu M, Mai R, Gozzo F, Francione S, Tassi L, Lo Russo G, Antiga L, Ferrigno G (2014) Multi-trajectories automatic planner for stereoelectroencephalography (SEEG). In: IJCARS

27. Tokuda J, Fischer GS, Papademetris X, Yaniv Z, Ibanez L, Cheng P, Liu H, Blevins J, Arata J, Golby AJ, Kapur T, Pieper S, Burdette EC, Fichtinger G, Tempany CM, Hata N (2009) OpenIGTLink: an open network protocol for image-guided therapy environment. Int J Med Robot 5(4):423-34

28. Horn BKP (1987) Closed-form solution of absolute orientation using unit quaternions. J Opt Soc Am 4(4):629-642
29. R Core Team (2013) R: a language and environment for statistical computing. R Foundation for Statistical Computing, Vienna, Austria. http://www.R-project.org/

30. Ivanov M, Ciurea AV (2009) Neuronavigation, principles, surgical technique. J Med Life 2(1):29-35

31. Paul P, Morandi X, Jannin P (2009) A surface registration method for quantification of intraoperative brain deformations in imageguided neurosurgery. IEEE Trans Inf Technol Biomed 13(6):97683

32. Comparetti MD, Beretta E, Kunze M, De Momi E, Raczkowsky J, Ferrigno G (2014) Event-based device-behavior switching in surgical human-robot interaction. In: Proceedings of the 2014 IEEE international conference on robotics and automation (ICRA, Hong Kong), pp 1877-1882

33. Jannin P, Morandi X, Fleig OJ, Le Rumeur E, Toulouse P, Gibaud B, Scarabin JM (2002) Integration of sulcal and functional information for multimodal neuronavigation. J Neurosurg 96(4):713-23

34. Dumpuri P, Thompson RC, Cao A, Ding S, Garg I, Dawant BM, Miga MI (2010) A fast and efficient method to compensate for brain shift for tumor resection therapies measured between preoperative and postoperative tomograms. IEEE Trans Biomed Eng 57(6):1285-96 